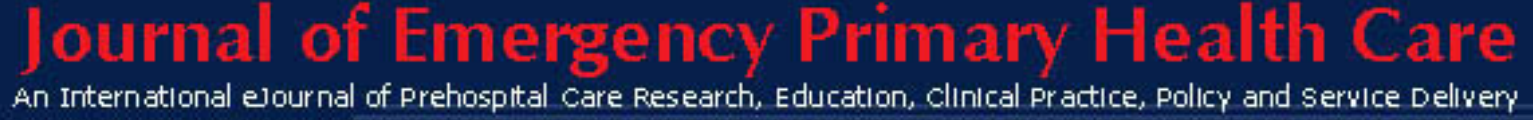

ISSN 1447-4999

\title{
EDITORIAL
}

\section{Closing the knowing-doing gap}

Article No. 990093

\begin{abstract}
Dr. Heather Buchan - Chief Executive Officer, National Institute of Clinical Studies, Australia
Professor Chris Baggoley - Director Emergency Medicine, Royal Adelaide Hospital, Board Member National Institute of Clinical Studies, Australia
\end{abstract}

There is a significant gap between what is known from the best available research and what actually happens in clinical practice. Recent studies from the United States and from primary care settings in the Netherlands $(\mathbf{1}, \mathbf{2})$ suggest that 30 to 40 per cent of patients do not receive treatments of proven effectiveness and that 20 to 25 per cent have treatments that are unnecessary or potentially harmful. A recent publication from the National Institute of Clinical Studies (3) highlights a selection of specific evidence practice gaps that exist in Australia and identifies the impact that these "knowing-doing gaps" have on people's health and well being.

The reasons why evidence isn't translated into practice are varied and there may be barriers to evidence uptake at the patient, clinician, team, organization or system level. There is no single strategy that will work in all situations to improve evidence uptake. Strategies aimed at improving knowledge or skills of individual clinicians will not be helpful if the core problem relates to the way in which work flows and processes operate within an organization. Ways to improve uptake of research evidence are most successful if the barriers to implementation in specific areas are understood, and interventions tailored to overcome these barriers are applied.

In comparison to the amount spent on basic health care research, the amount spent on investigating and understanding ways to improve use of research is small, particularly in the public sector. Where there is a commercial interest in promoting uptake, such as with pharmaceuticals, specific companies and the industry in general put a substantial investment in promoting use of relevant products. This type of investment is not generally made in areas where research findings have less immediate commercial application, and there has been a reliance on more traditional medical ways of knowledge diffusion and uptake.

In 2001, the Australian government recognized the need to more actively promote the use of research findings in health care by;

- raising awareness of the important gaps between what is known, from the best available research, and what is actually done in day-to-day practice; and,

- supporting health professionals to understand and overcome the barriers to applying evidence within Australian health care settings. 
Funding provided by the Australian government is used to;

- undertake national implementation projects in areas where there are important evidence practice gaps,

- trial different methods for changing practice and improving uptake of evidence,

- increase and develop national expertise in implementation, and to

- produce and promote resources that will help clinicians improve use of evidence in clinical practice.

The Institute has a wide brief and limited resources. It has chosen to initially undertake implementation projects in a small number of clinical priority areas that span a range of settings, issues, disciplines and challenges for evidence uptake. Further deciding factors in consideration of specific clinical priority areas included the willingness of clinical groups and other relevant organisations to work in partnership with the Institute to design and implement changes to care, and to trial different approaches to improve evidence uptake.

One of the first projects tackled by the Institute in 2002 was aimed at helping to improve the use of evidence in emergency departments. This involved teamwork with the Australasian College for Emergency Medicine, the Australian Emergency Nurses' Associations, the state health departments and 47 hospitals from around Australia, using collaborative methods developed by the Institute for Healthcare Improvement in the United States. The common focus for improvement was to reduce time to analgesia, in response to the frequency of this issue being reported by emergency staff as a concern in $50-80 \%$ of presentations, with evidence to suggest under-treatment. Participating hospitals also chose to work on improving other indicators which were of concern locally, such as time to thrombolysis, pathology turnaround time and referral to specialist units.

\section{NICS organized;}

- collaborative and various face to face meetings and teleconferences

- design and management of a website as a provision for participating hospitals to post their data and information about various protocols and strategies which they had employed to change performance

- a variety of national and international experts to contribute ideas

- provision of a number of resources such as evidence reviews, culture and patient perception surveys and,

- evaluation of key aspects of the collaboration.

Thirty four hospitals showed an improvement in time to analgesia, with seven achieving more than a 50\% reduction in the median time to analgesia. A study is currently underway to determine whether improvements have been maintained over time. Further information on results, strategies for change, stories from participating hospitals and various resources are available on the NICS website: www.nicsl.com.au.

Many of the participants in this project were keen to continue to be involved in joint initiatives and NICS is now trialling a "Community of Practice" in emergency Care. A Community of Practice is a voluntary network of people who share information, build on existing knowledge, develop expertise and solve problems for a common purpose driven by the interest of the community involved. Community of Practice models in sectors other than health, have enabled rapid spread of knowledge and innovation. The first project to be launched within the NICS Emergency Care Community of Practice is an initiative aimed at improving care at the Emergency Department: Mental Health interface. Over a 12 month 
period participants in the project will aim to ensure that $90 \%$ of people presenting to the emergency department with a mental health problem, are discharged, transferred or admitted within 4 hours, in an effort to reduce the "Did not wait" rate to $3 \%$ or less, and that unscheduled representations for people with mental health problems within 72 hours is reduced by $50 \%$.

NICS is undertaking other major implementation projects aimed at improving diagnosis and management of heart failure and management of pain in cancer care. A major project aimed at preventing deep vein thrombosis in hospitalised patients is planned for 2005.

Further information about these projects and a range of resources that have been produced from work to date are available on the NICS website. A primary care resource guide summarises the resources that may be of interest to people working in primary care. These include the Cochrane Library (NICS has negotiated a national license on behalf of the Australian Government which provides free access to the Library for all Australians) and a training guide to help people make best use of the Library; a range of resources relating to heart failure, including links to the four highest quality publications designed for patients with heart failure; a pressure ulcer resource guide, and a number of literature reviews. Information on the outcomes of projects funded through an initial NICS targeted grants program is also available on the website - examples of potential interest to readers of JEPHC, include a study on reducing the inappropriate use of cervical spine X-rays in alert and stable blunt trauma patients (led by Dr Kevin Chu from the Royal Brisbane Hospital in Queensland); a report on optimizing the use of pethidine in hospital emergency departments (from Ms Karen Kaye of the NSW Therapeutic Assessment Group) and an investigation on ways to implement the Canadian CT Head Rule (from Dr Paul Lamberth of the Canberra Hospital). Presentations from visiting international experts who have conducted public talks and workshops for NICS are also available on the website - these include a talk on how to implement change in primary care practice (from Professor Richard Grol of the Netherlands one of the leading world researchers in this area) - and a presentation on brokerage between researchers and health care decision makers from Professor Jonathan Lomas, Executive Director of the Canadian Health Services Research Foundation.

The Institute has also invested in a longer term capacity building program, by developing evidence implementation Fellowships, with five Fellowships being awarded in the first year of this program. Among the first Fellows appointed was Associate Professor Steven Doherty from Tamworth, who aims to improve adherence to evidence based guidelines on asthma within the New England region of New South Wales.

The Institute has a regular newsletter to inform people of new opportunities and projects; resource posting on the NICS website, and upcoming workshops or talks. Readers of JEPHC who wish to know more about NICS' activities can subscribe to the newsletter by visiting the NICS web page and requesting a subscription. 


\section{REFERENCES:}

1. Grol R. Successes \& failures in the implementation of evidence based guidelines for clinical practice. Medical Care 2001; 39 (suppl 2): pp 46 - 54.

2. McGlynn EA, Asch SM, Adams J et al. The Quality of Care delivered to adults in the United States. N Engl J Med 2003 348; 26: 2635-45.

3. National Institute of Clinical Studies: Evidence Practice Gaps Report Volume 1: http://www.nicsl.com.au/knowledge_reports_detail.aspx?view=15 . Accessed July 2004. 